\section{Participatory community-based arts for older people living with dementia in the community: how are they evaluated? A scoping review}

\author{
Azam Bazooband, \\ Helen Courtney-Pratt, \\ Kathleen Doherty \\ Wicking Dementia Research and \\ Education Centre, University of \\ Tasmania, Hobart, Australia
}

\begin{abstract}
Although the interests in participative arts for people living with a dementia has increased over the last decade, what is yet to be reviewed is how participatory community-based arts activities for this group of people are evaluated. The overall aim of the following scoping review is to understand the scope of measurement/evaluation methods/approaches used in studies that recruited participants with dementia from the community (not from health/clinical or residential aged care settings or nursing homes) and delivered community-based participatory arts activities/programs (not art therapy programs) to them.
\end{abstract}

The methodological framework by Arksey and O'Malley (2005) for undertaking a scoping review article was applied to this study. Collation, summarizing and reporting the results was carried out considering the research questions. 7 articles met inclusion/exclusion criteria published from 2013 to 2020. The type of arts activities included co-designs and co-creation of various types of arts $(\mathrm{n}=1)$, museum visiting and art-making activities $(n=4)$, artistic education-based program $(n=1)$, group singing $(\mathrm{n}=1)$.

This scoping review shed light on the paucity of research in which older people living with dementia were recruited from the community (not healthcare/clinical settings) to participate in participatory community-based art activities. Also, the results revealed that evaluation of participatory community-based arts activities for older people living with dementia in the community should include methods/techniques to get a deeper insight into the participants' values and perspectives and the social interaction benefits of such programs.

\section{What is known about this topic and what this paper adds}

\section{What is known about this topic?}

- The number of people living with dementia is increasing globally, and people living with dementia tend to live in the community, which is attributable to advantages in terms of preserving a sense of belonging, security and familiarity, as well as a sense of identity and autonomy.

- Participatory community-based arts activities without being constituted as a therapy aim to promote health and wellbeing in those living with dementia.

- Participatory community-based art activities - defined broadly as a series of art activities in which individuals participate actively in community settings and are mainly provided by artists with no particular background in health education - can foster social inclusion and community involvement in those living with dementia in the community.

\section{What this paper adds?}

- Few studies recorded the effect of participatory community-based arts activities on people living with dementia in the community and included social engagement as an outcome measure.

- Most results from research on art-based approaches for people living with dementia in the community are more provisional than compelling.

- More rigorous metrics are required to assess the outcomes of participatory community-based arts programs (particularly social engagement aspects) for those living with dementia in the community.

\section{Introduction}

Global life expectancy has increased since 1950 by 22.4 and 23.7 years for men and women respectively. ${ }^{1}$ An increase in the prevalence of dementia is one consequence of population ageing. ${ }^{2}$ The number of people living with dementia is projected to reach 75 million by 2030 and around 132 million by $2050 .^{3}$ As a consequence, the World Health Organization (WHO) recognized dementia as a health-care priority for the coming decades. ${ }^{4}$

People living with dementia experience functional decline, which negatively
Correspondence: Azam Bazooband, Wicking Dementia Research and Education Centre, University of Tasmania, Room 417H,

Medical Science 1, Hobart CBD Campuses, Australia.

E-mail: azam.bazooband@utas.edu.au

Key words: Dementia; participatory art; community-based art; scoping review; arts assessment tools; participatory community-based arts.

Acknowledgments: the authors would like to thank the University of Tasmania and Wicking Dementia Research and Education Centre for funding to support this review, which was the part of a $\mathrm{PhD}$ project.

Funding: this research was part of a $\mathrm{PhD}$ project supported by the University of Tasmania Graduate Research Scholarship.

Conflict of interests: the authors declare no potential conflict of interests.

Received for publication: 7 November 2020. Accepted for publication: 14 January 2021.

This work is licensed under a Creative Commons Attribution-NonCommercial 4.0 International License (CC BY-NC 4.0).

${ }^{\circ}$ Copyright: the Author(s), 2021

Licensee PAGEPress, Italy

Geriatric Care 2021; 7:9470

doi:10.4081/gc.2021.9470

impacts physical and cognitive capacity. 5,6 Because of the progressive detrimental effects on memory, cognition, language, behavior, planning, motivation, and judgement: ${ }^{7}$ people with dementia may also experience social consequences which deter them from remaining active in their community. The stigma associated with dementia is a major issue,${ }^{8}$ and may result in intentional and non-intentional exclusion of individuals living with dementia from participating in mainstream society. This has negative implications for individuals living with dementia and society more generally. ${ }^{9}$

The majority of people living with dementia continue to live within their communities, ${ }^{10}$ often cared for by a family member. Ongoing social inclusion for both the person living with dementia and their caregiver offers tangible benefit given the relationship between social connection, social support and overall health. ${ }^{11}$

Broader community benefits can also result, for example, support for the continued participation of people with dementia in community activities can lead to generally enhanced understanding of dementia, 
which can have a significant effect on wellbeing of people living with dementia and their families. ${ }^{12}$

Various types of community engagement activities are designed to ensure people living with dementia, their families and caregivers, are supported to retain their connection to the community. Such activities include co-creation programs, ${ }^{13}$ reminiscence programs, ${ }^{14}$ leisure activities, ${ }^{15}$ physical and exercise activities, ${ }^{16}$ and community gardening activities, ${ }^{17}$ to name a few.

Among various opportunities to contribute meaningfully to community engagement, there is growing evidence that community-based arts programs contribute to well-being and community improvement. ${ }^{18,19}$ Additionally, the social advantages of taking part in participatory arts activities include peer support, collaborative interactions with art facilitators and enhanced social skills. ${ }^{20,21}$ For instance, Burnside et al. (2017) demonstrated that participation in art museum activities (including an art gallery tour combined with art-making classes) for people with dementia provided the opportunity for recreation, socialization and respite. However, the study's participants have acknowledged such programs can also have more profound personal and relationship benefit such as relationship affirmation, normalization and personal growth. ${ }^{22}$ Activities such as group singing were reported to be likely to positively contribute to the experience of the relationship between couples where one partner is living with dementia. ${ }^{23}$

Participatory art activities can be broadly described as a set of art activities in which individuals actively participate and are chiefly delivered in community settings by artists with no specific health education background. ${ }^{24}$ The objectives and goals of participatory arts initiatives differ from the practice of art therapists who are normally health care workers/professionals working in healthcare environments (hospitals or hospices), and seeking to ameliorate specific conditions. ${ }^{25}$ Rather than clinical outcomes as emphasized in art therapy, participatory arts activities are primarily a means of engaging older people- including those with dementia- within the community. Given this outcome measures, the evaluation approach to assess the outcomes of each initiative should be specifically chosen to reflect such differences. ${ }^{26}$

The beneficial and instrumental function of arts activities and their capacity to improve participants' health, wellbeing or quality of life have been the primary focus. ${ }^{27-31}$ For older adults, including those living with dementia, sometimes measures of physical or cognitive function have been used to assess the outcomes. ${ }^{32,33}$

Although participatory arts initiatives are well known, both in practice and in the literature, the effectiveness of activities' content is sometimes overemphasized, and relatively little consideration has been devoted to the design and evaluation of such activities and their health benefits for older adults living in the community. ${ }^{25}$ This supports the increasing need for relevant evaluation methods/approaches to assess community-based participatory arts and potentiate results of social engagement for people living with dementia. ${ }^{34}$

Additionally, it is crucial for every program (including participatory communitybased arts programs) to have a variety of evaluation instruments/approaches appropriate to the needs of the participants (such as those living dementia) which measure/ assess the impacts across the right outcome measures or evaluation approaches. ${ }^{35,36}$ Otherwise, the impact of a program can be overlooked or exaggerated without choice of appropriate tools. So, the choice of relevant outcomes, and robust tools to measure those, is a vital stage in designing the evaluation of activities and programs intended for older people, particularly those living with dementia. ${ }^{37,38}$

This raises the question of how participatory community-based art activities delivered to those living with dementia in the community are evaluated and whether there are gaps regarding the range of outcome measures or evaluation methods/approaches applied to specifically assess the success of such activities for people living with dementia in the community. It needs to be mentioned here that, although very recent literature ${ }^{39}$ has specifically explored the overall impact and holistic benefits of participatory community-based arts activities for people with early to moderate stages of dementia, a scoping review has not yet been applied to explore an overview to participatory community-based art programs specifically for older people living with dementia in the community (not those living in health/clinical or residential aged care settings or nursing homes) and how they are assessed; however, it may be useful to highlight the paucity of research in participatory community-based arts for those living with dementia in the community and to identify limitations in evaluation methods/approaches in such participatory arts implementation.

Influenced by previous literature and knowledge gaps, and as the first phase of $\mathrm{PhD}$ research, this scoping review aims to explore the scope of evaluation approaches used in studies examining the impact of par- ticipatory community-based arts (excluding art therapy) delivered to older people living with dementia in the community (excluding the studies in which participants were recruited from health/clinical settings/ residential aged care settings, and nursing homes). So, the scoping review has the following objectives:

- To offer an overview of the different participatory community-based arts implemented for people with dementia in the community.

To explore: i) the measured/evaluated content of participatory communitybased arts for those living with dementia in the community; ii) the evaluation approach used to assess the outcomes of participatory community-based arts for those living with dementia in the community; iii) the limitations regarding evaluation methods used to assess the outcomes of participatory communitybased arts for those living with dementia in the community.

This study's results may provide an opportunity for future researchers to identify the limitations of available evaluation methods/approaches for assessing the outcomes of participatory community-based arts programs for older people living with dementia in the community and consider the implications of designing their activities to include participants with dementia. The results may also raise the importance of approaches to capture the perceived effectiveness of such programs more broadly, considering their initial aims and goals.

\section{Materials and Methods}

A scoping review is a tool to review, explore and map a wide range of research activity of various methods and quality and to assess the breadth/depth and gaps or opportunities of a field. ${ }^{40,41}$

The Methodological Framework underpinned by Arksey and O'Malley (2005) was adopted in this study which encompasses 5 stages: i) defining the research question to be addressed; ii) identifying relevant/potential studies; iii) study selection; iv) charting the data; and v) collating, summarizing and reporting the results. ${ }^{42}$

\section{Determine the purpose}

The purpose of this study was to identify the measurement tools used to evaluate arts activities' outcomes delivered within the context of community for older people living with dementia. The scope of participatory art activities was defined as those types of arts-focused activities in which the 
main intents were getting people engaged in participatory art activities and being socially active or promoting health and wellness and designed for and delivered to older people residing in the community rather than health or clinical settings. The arts activities were diverse and included, but were not limited to music, dance, theatre, creative activities, painting, and drawing.

Considering the existing overlap in the literature regarding the concept of participatory community-based arts activities and art therapy, we elected to use search logs including art therapy at the first stage of our search and in the next step apply explicit inclusion and exclusion criteria to titles/abstracts and then to full-text articles. This search strategy helped the researchers to ensure that those arts projects run as community-based arts activities, but titled art therapy were not inadvertently excluded from the search.

\section{Identify potential studies}

Various databases were searched including PubMed, PsychINFO, ProQuest, Scopus, CINAHL, MEDLINE, Web of Science, Embase, and Social Sciences Citation Index. The search terms were developed with the assistance of a research librarian. The search logs included aged OR aged [Text Word] OR elderly [Text Word] OR senior [Text Word] OR older people [Text Word] OR geriatric [Text Word] AND art therapy [Mesh term] OR dance therapy [Mesh term] OR music therapy [Mesh term] OR sensory art therapies [Mesh term] OR art therap* [Text Word] OR music therap* [Text Word] OR dance [Text Word] OR music [Text Word] OR singing [Text Word] OR creative arts [Text Word] OR theatre therap* [Text Word] OR participatory arts [Text Word] OR arts activities [Text Word] OR community arts activities [Text Word] OR community-based arts [Text Word] AND Dementia.

\section{Screen and select studies}

Our inclusion criteria were: i) primary research study; ii) included aspects of participatory arts activities; iii) intended to be for older adults living with dementia; iv) arts activities for older people living in the context of the community (both selective attendance and invited by researchers); v) research published in English within the last 10 years from available international literature; vi) qualitative, quantitative, and mixedmethod studies. Our exclusion criteria were: i) studies without any evaluating methods to define outcomes; ii) audience participation or non-participatory arts activities; iii) review articles; iv) art therapies, arts therapy is defined as the clinical use of multi-art forms - including but not limited to music, drama, and visual arts to accomplish individualized goals by the art therapists not the artists or art facilitators, within a therapeutic relationship; ${ }^{43} \mathrm{v}$ ) the studies in which the participants were recruited from aged care facilities, nursing homes, aged centers, clinics, and hospitals; vi) all studies which were not for older people with dementia. Also, the studies in which participants were recruited from health care settings have been removed from consideration; however, the arts activities which were held in healthcare settings have not been eliminated if the participants were recruited from the community rather than the specific health care setting.

The rationale of excluding the studies where recruitment of older people living with dementia had not occurred in the community while the activity/intervention occurred in the community was to highlight the paucity of studies in the field of art and dementia in which participants were community-dwellers living with dementia and were recruited from the community not aged care centers, health clinics or nursing homes.

The titles/abstracts were initially screened, and further screening was conducted on the full texts and a total of 7 studies (7 published articles) were included for data extraction (Figure 1). The list of all studies can be found in Table 1. The McMaster critical appraisal tools (McMaster Critical Review Form - Qualitative Studies (Version 2.0) and McMaster Critical Review Form Quantitative Studies ${ }^{44}$ were used to appraise the studies. Being freely available as well as their appropriateness for various research designs motivated the authors to choose these tools for this scoping review study. Differences and conflicts in the appraisal results were further discussed until consensus was reached between the authors.

All selected qualitative studies met the following criteria; the purpose has been stated clearly with a relevant literature review, the methods were congruent with the study purpose and theoretical perspective of the research, the study design was appropriate to the research question, with sample size, data collection, and data analysis described in detail and the conclusions were appropriate given the study findings.

Also, all selected quantitative studies had a clear purpose, relevant background literature review, well-described study design, containing details on sampling strategies. Furthermore, the outcomes area and evaluating methods were reported, and the studies had a clear description of the intervention, statistical analysis was reported in the results, conclusions and implications were appropriately described based on the study methods and results. The quality of mixed-method studies also was assessed against the parameters appropriate to methods used as described above.

\section{Collating, summarizing and reporting the results}

The extracted data were reviewed to determine the most suitable method for summarizing the findings. A content analysis was conducted to further analyze some concepts, including how the arts activities was conceptualized by the authors and the content of arts activities applied in the study, in order to synthesize this data into categories.

\section{Results}

All included participatory communitybased arts activities' characteristics that emerged from the qualitative, quantitative and mixed-method studies are summarized in Table 1, including the design, types of arts activities, the assessed/evaluated content, evaluation methods, outcomes and limitations regarding applied evaluation methods.

\section{Types of arts programs}

All 7 articles which met inclusion and exclusion criteria were published from 2013 to 2020. The types of arts activities included co-design and co-creation of various types of arts $(n=1)$, museum visiting and art-making activities $(n=4)$, various artistic education-based program $(n=1)$, and group singing $(n=1)$.

Participants' consent (both written and verbal) was sought in all studies.

\section{Museum-based activities}

Among the four museum-based studies, two had quite bigger population size (around 100) and the other two ${ }^{22,28}$ had smaller population size (less than 50). Except for one study, ${ }^{45}$ caregivers (formal caregivers, family members or friends) were included as participants. The rationale for excluding caregivers in the study by Camic et al. (2019) was to assess the feasibility of the activity without the presence of family members or near friends. Also, in all four studies, people with mild to moderate dementia level were included. As part of the recruitment strategy, organizations such as Alzheimer associations were involved in all museum-based studies, along with referrals from local physicians ${ }^{22}$ and newspaper advertisements. ${ }^{46}$ Except for one study which had a wait-list control group, ${ }^{46}$ all other gallery-based studies were non-controlled studies. The shortest study consisted of eight weeks period ${ }^{28}$ and the rest were 
longer interventions; up 12 months $^{22,45}$ or two years. ${ }^{46}$ The art viewing in three studies $^{22,28,46}$ was accompanied by art-making sessions, and only in one study, ${ }^{45}$ the museum object handling sessions were followed by non-memory-related open discussions rather than art-making sessions. Except for one study, ${ }^{28}$ the museum staff or art educators were trained according to standards such as visual thinking strategies (VTS), ${ }^{22}$ TANDEM training manual, ${ }^{46}$ and dementia awareness training ${ }^{45}$ to work with and communicate with people living with dementia.

\section{Mixed types of arts activities}

Two studies ${ }^{13,47}$ used mixed types of arts activities either for educational purpose ${ }^{47}$ or for involving older people with dementia in co-designing and co-creation programs. ${ }^{13}$ Only in one study ${ }^{13}$ the carers were included in the intervention. Both studies had a small sample size with no control group; however, only in one study ${ }^{47}$ the small sample size and composition was reported as a limitation. One study ${ }^{13}$ lasted for two months while the other was 4 months. ${ }^{47}$

Both studies were set up in series workshops but with different approaches and using various types of arts. In the study by Tsekleves et al. (2020) participants and research team joined in a creative co-design phase, utilizing a range of artistic and tactile materials (Table 1). However, in the other study by Ullán et al. (2013) workshops consisted of viewing materials of diverse artists' works, commenting on them, fol- lowing by conducting specific personal cyanotype work.

\section{Singing}

Only in one study, ${ }^{23}$ group singing was used as an intervention for 17 couples where one had dementia (mild to moderate) and were still living in their own homes. Interestingly, there were differences in the types of singing activities; people living with dementia and their spouses engaged in either time-limited or ongoing singing sessions combined with singing and art viewing ending in a performance, or with music-making sessions, or with a movement group; which reportedly provided researchers with a unique opportunity to compare the results from various intervention groups.

\section{PRISMA 2009 Flow Diagram}

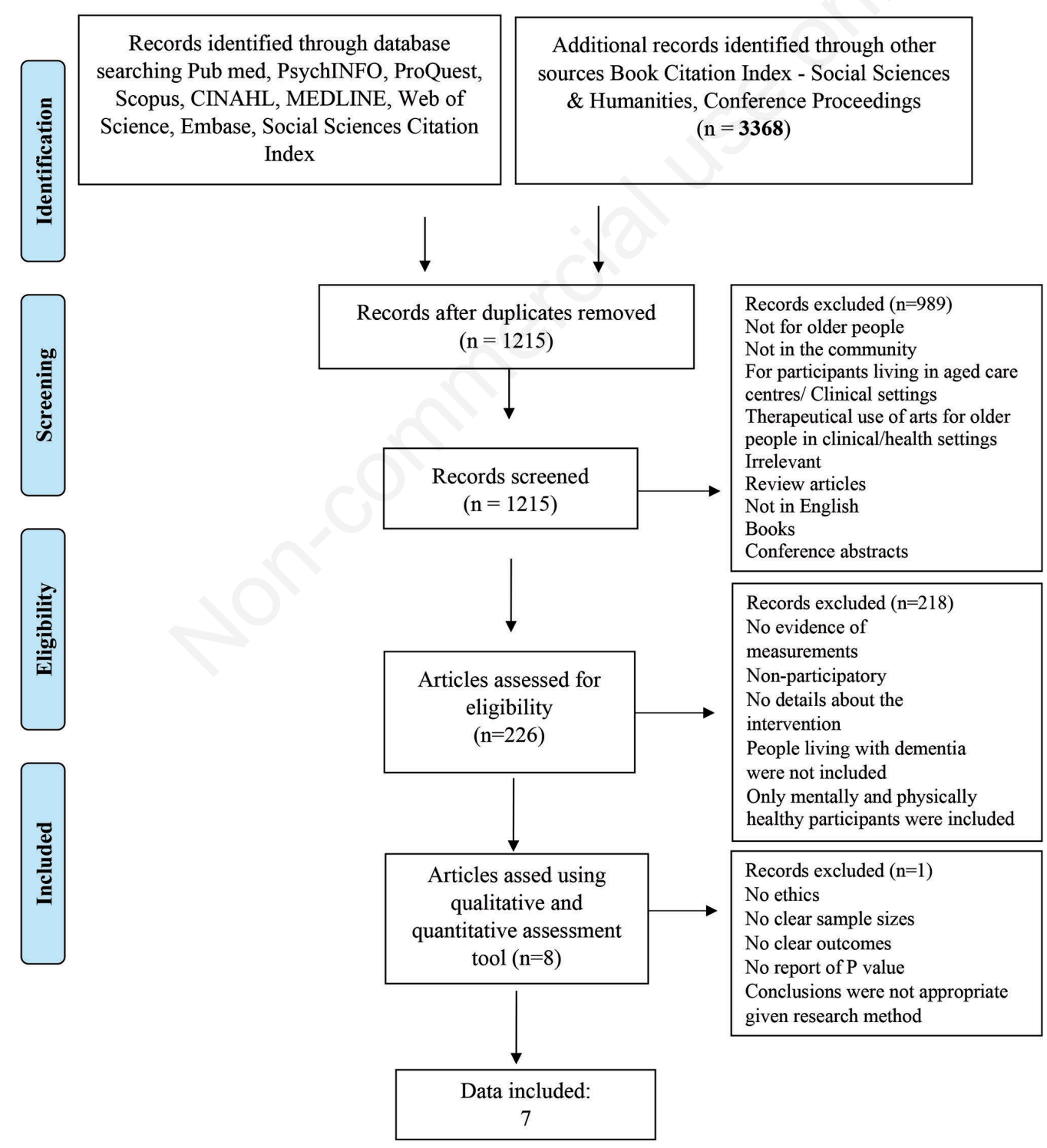

Figure 1. Search results. 


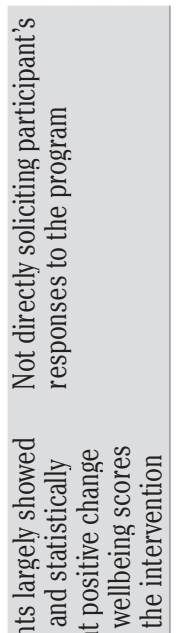

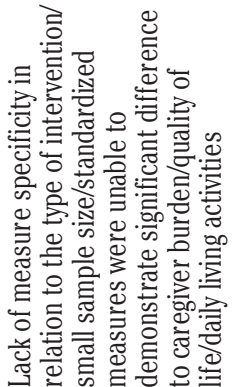

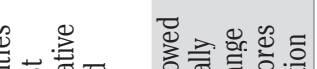
定 $\dot{1}$

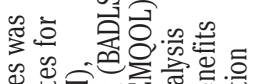

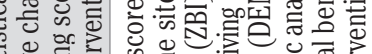

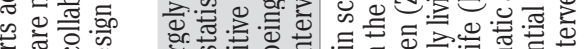

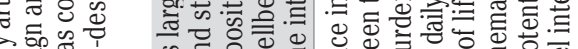

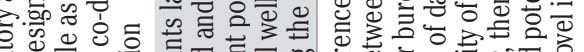

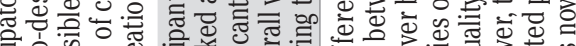

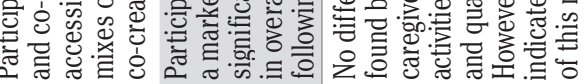

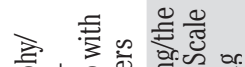

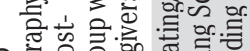
응 항응휴 卷 응

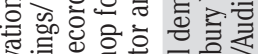

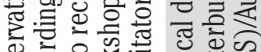

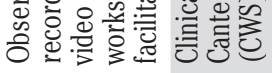

\section{요요}

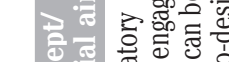

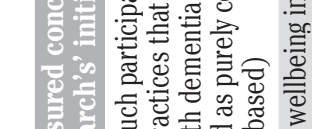

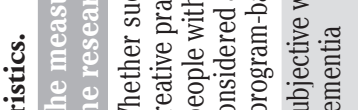



吾

E

瀶

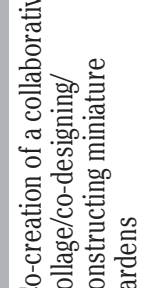

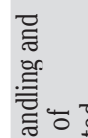

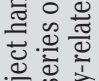

응 of

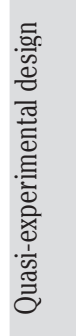

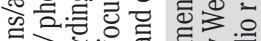

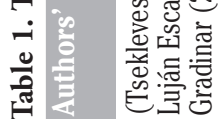

\section{龵 蒙}

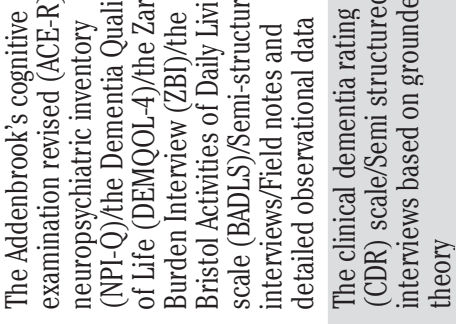

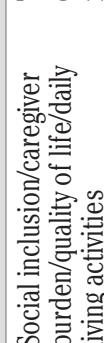

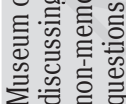

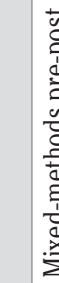

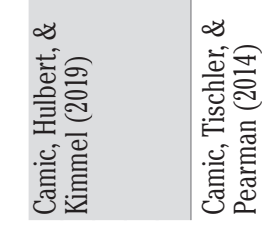

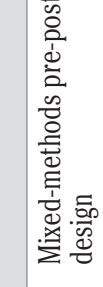

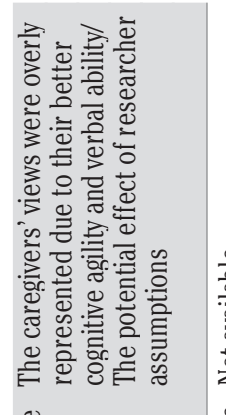

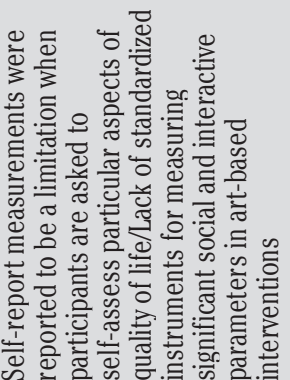

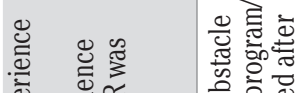

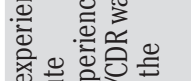

들

紫

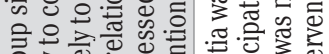

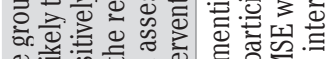

至.心

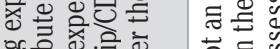

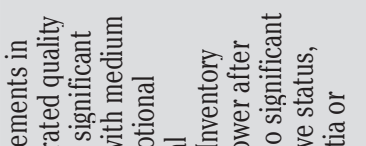

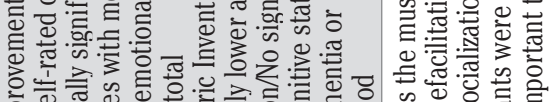

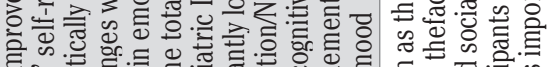

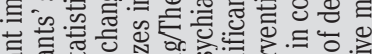

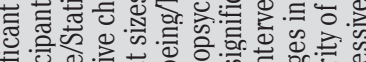

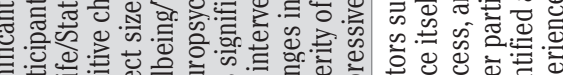

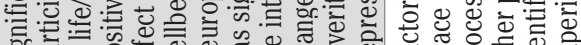

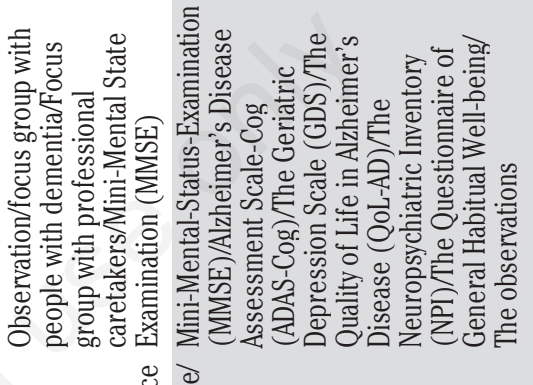

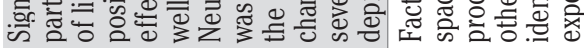

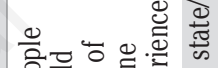

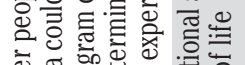

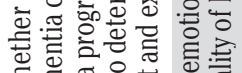

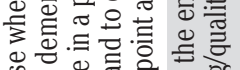

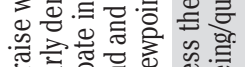

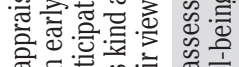

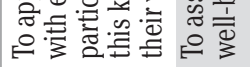

응

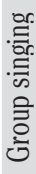

.

ฮิ

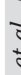

苟

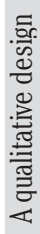

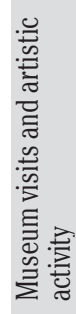

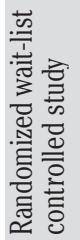

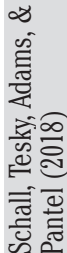

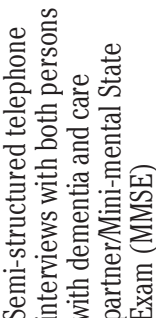

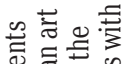

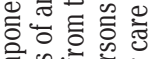

宕总

过 항

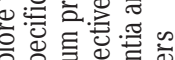

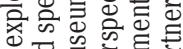

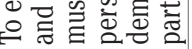

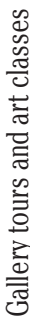

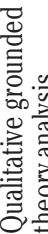

포웜ㅇ

,

萬 


\section{Evaluated content}

Program intent and outcome assessed varied. While in one study ${ }^{13}$ the main focus was on the nature of the program and to assess how to leverage the advantages of cocreation or co-design in creating a workspace layout that may help carers and support staff when designing activities for people living with dementia; in another three studies ${ }^{22,23,47}$ the participants' experiences were the main outcome evaluation. The rationale for choosing the evaluation methods was different in each study. In one study ${ }^{22}$ it was based on the researchers' assumption that obtaining knowledge of the participants' experiences would provide valuable details for the continued growth, progress and assessment of those programs. However, the in-depth analysis of participants' experience in two other studies ${ }^{23,47}$ was conducted to determine whether these individuals might be able or willing to engage in the system and how such interventions would add to their quality of life, or to assess how such programs might be beneficial for those living with dementia as well as their partners. Being more focused on participants' benefits, subjective wellbeing was assessed in two studies, ${ }^{45,46}$ with the aim of better understanding of cognitively stimulating, creative, socially rewarding and engaging activities for people living with dementia, without depending on reminiscence, past experience or memory. Also, other potential outcomes such as social inclusion, caregiver burden, and daily living activities were only assessed in one study ${ }^{28}$ based on integrating three different theoretical insights (the constructivist museum model; ${ }^{48}$ uncommitted potentiality of change, ${ }^{49}$ and positive influences of arts on health promotion ${ }^{50}$ ). Quality of life was assessed in two studies. ${ }^{28,46}$

Considering the assessed content and aims of the included studies, results of this scoping review revealed that in only two studies $^{23,28}$ social inclusion was specifically targeted. However, even if not basically/initially identified, the social engagement aspects of participatory arts programs were acknowledged in another three studies. $13,45,47$

\section{Evaluation approaches}

\section{Cognitive assessment tools}

Except for one study ${ }^{13}$ which did not include any cognitive assessment tools, various evaluation methods were implemented for various purposes such as assessing the level of participants' impairment for inclusion purposes, ${ }^{22,23,28,45,47}$ and to use as a measure of functional decline in people with dementia before and after the intervention; ${ }^{46}$ among which mini-mental state exam (MMSE), was the most common, either administered as the sole cognitive impairment evaluation approach ${ }^{22,47}$ or in combination with other cognitive assessment tools such as Alzheimer's disease assessment scale-Cog (ADAS-Cog). ${ }^{46}$ Also, severity was determined using other evaluation methods/ approaches such as the clinical dementia rating (CDR) scale in the study by Unadkat et al. (2017). Only in one study, ${ }^{28}$ caregivers' perspective was assessed using Addenbrook's cognitive examination revised version (ACE-R) $)^{51}$ along with the neuropsychiatric inventory (NPI-Q) (an evaluation approach used by carers to identify neuropsychiatric symptoms in older adults $)^{52}$ as proxy.

\section{Tools to assess wellbeing}

Two different scales were administered as pre-test, post-test to assess the emotional ${ }^{46}$ and subjective wellbeing ${ }^{45}$ of participants. Although both scales were self-rating and based on pictorial and visual response system, the questionnaire of general habitual well-being used by Schall et al. (2018) was shorter with seven faces ranging from very happy to very sad. Moreover, the Canterbury wellbeing scale $(\mathrm{CWS})^{53}$ was implemented by Camic et al. (2019) with the rationale that this scale has been designed to be used by people living with dementia, having advantages such as being visual, easy-to-administer, providing minimal distraction, not being unpleasant and suitable for use in various community settings. ${ }^{45}$ Using the standard well-being scales in both studies, researchers were able to demonstrate marked, significantly positive change in participants' wellbeing following the intervention. The only difference was that in the study by Camic et al. (2019) detailed results showed larger positive increases in wellbeing in early stage participants compared to those in mild stage of the condition.

\section{Tools to assess quality of life}

To assess quality of life, the dementia quality of life (DEMQOL-4) questionnaire ${ }^{28}$ and quality of life in Alzheimer's disease (QoL-AD) questionnaire ${ }^{46}$ were used. Both scales were administered as self-assessment pre-post-test tool. Although the intervention group demonstrated significant improvement in self-reporting quality of life (QoL-AD) in the study by Schall et al. (2018), self-assessed quality of life in the study by Camic et al. (2014) remained stable after the intervention.

\section{Other standard evaluation methods}

Four more standard measurement tools were used in two studies $(28,46)$ for various purposes. Two of the scales were assessed by the carers ${ }^{28}$ including the Zarit burden interview (ZBI) (which is a 22 item scale to assess the severity of caregiver perceptions of burden or stress in caring for people living with dementia), and the Bristol Activities Of Daily Living Scale (BADLS) ${ }^{24}$ to assess the ability of people living with dementia to do daily activities. Although no statistically significant difference in scores was found between the sites for caregiver burden (ZBI) and activities of daily living (BADLS), there was a marginal movement towards reducing the carers burden over the duration of the study, which was reinforced more clearly by thematic studies.

The other two standard scales were administered as pre-post-tests ${ }^{46}$ to assess depressive symptoms of the participants and neuropsychiatric symptoms of people with dementia. Although the geriatric depression scale $(\mathrm{GDS})^{46}$ was considered suitable for assessing depressive symptoms in older adults, the neuropsychiatric inventory $(\mathrm{NPI})^{46}$ was a more dementia specific scale, designed to assess 12 neuropsychiatric symptoms usually found in dementia such as delusions, agitation, depressed mood, anxiety or apathy. Both scales demonstrated beneficial effects of the intervention on depressive symptoms and dementia related behavior (particularly agitation and apathy).

\section{Qualitative evaluation methods}

A broad spectrum of qualitative assessment tools was used in all studies, even as the sole method of collecting data ${ }^{13}$ or as a mixed methods approach with different quantitative scales..$^{22,23,28,45-47}$ All studies were consistent in applying at least one qualitative evaluation approach for collecting data to assess the benefits of interventions.

In 5 of the studies, ${ }^{13,22,23,28,47}$ different types of interview were undertaken to collect in-depth information from the participants. Interview sessions were conducted either as focus group interviews with facilitators and carers, ${ }^{13}$ focus groups with people with dementia as participants and professional carers, ${ }^{47}$ or as semi-structured interviews with people with dementia and carers. ${ }^{22,23,28}$

Collecting data through observations and taking field notes was the most popular qualitative data collection method following interview, used in four studies. ${ }^{13,28,46,47}$ The observation process conducted in the study by Schall et al. (2018) was different from the rest of studies, as it was based on using an observation instrument called ' $C O D E M{ }^{54}$ which is designed specifically for assessing communication behavior in people living with dementia.

Also, in some studies further data collection from the actual intervention sessions/workshops were conducted through audio recordings, ${ }^{13,45}$ video recordings and photography ${ }^{13}$ of each session. 


\section{Limitations regarding evaluation methods}

A wide range of limitations regarding evaluation methods/approaches and scales to assess the effectiveness of interventions were discussed in almost all included studies. For instance, some limitations were related to the lack of focus on the intervention such as lack of explicitly seeking answers from the participants related to the intervention ${ }^{45}$ or the lack of measure specificity in relation to the type of intervention. ${ }^{28}$

Also, in some studies, attention was called to the need for tailored standardized measurement tools to assess the target parameters such as social and interactive parameters ${ }^{46}$ as well as mood, communication, social engagement, and other salient outcomes $^{22}$ in arts-based interventions for people living with dementia; creation and validation of those scales was strongly recommended due to the lack of clear outcome evaluation approaches/methods. ${ }^{22}$

Moreover, in some cases the limitation was not due to the lack of suitable scale, instead it was related to the lack of application in dementia research. To illustrate, the visual analogue scale (VAS) was reported to be suitable yet neglected scale in dementia research. ${ }^{45}$ A paucity of visual/pictorial scales, was reported to be a limitation specially when seeking self-report from people living with dementia beyond a certain degree of cognitive impairment. ${ }^{46}$

Furthermore, some limitations were reported as the result of using qualitative design such as the potential effect of researcher assumptions and overly representing caregivers' points of view due to their better cognitive and verbal ability while conducting interview sessions. ${ }^{23}$ However, in another study. ${ }^{28}$ using thematic analysis of observations and field notes successfully demonstrated the benefits of the implemented intervention, while the results of standardized scales failed to support the same outcome.

\section{Discussion}

There are a range of examples of community-based art activities designed and delivered solely for older people with dementia, recruiting participants from aged care settings, including aged care, community mental health services and in-patient centres or delivering art activities in day centres recruiting participants from the same centres or continuing-care retirement community or in-patient centre. Although the research described in abovementioned studies delivered art activities in the com- munity, some might claim it couldn't be considered as a genuine community-based research as recruitment was limited to discrete areas; so, as far as community-based art activities are concerned, the results of this study indicated that there are only a few studies described as recruiting their participants from the general population rather than clinical/health care settings. ${ }^{13,22,23,28,45-47}$

Despite a large volume of literature being available in the domain of arts, few studies reported the impact of participatory arts activities on older community residents living with dementia and included social engagement as an outcome evaluation approach. ${ }^{45,55-57}$

This study confirms that that the characteristics of arts activities within the context of the community which determine that it is, in fact participatory community-based art, are not clear. Analysis of the literature on participatory arts activities in the community is complicated by the absence of a clear definition for community-based art programs. This result is also in line with previous studies..$^{25,33}$

Furthermore, results revealed that despite the perceived importance of participatory community-based arts activities for older people living with dementia, there remains a paucity of evidence in this field. This finding is consistent with that of Zeilig et al. (2014), who critiqued the participatory arts programs for people living with dementia and emphasized the necessity for more studies which take place over longer periods, for a broader spectrum of people living with dementia, and studies which utilize a variety of assessment processes.

Most results from research on art-based approaches for people living with dementia in the community are more indicative than compelling. This might partially be due to methodological limitations such as the absence of control groups, the choice of evaluation methods/approach, brief intervention times and limited sample sizes, or focusing on the long-term impact of arts projects. These results also, match those observed in earlier studies. ${ }^{31,46}$

The most obvious finding to emerge from the analysis is that, a very limited array of outcomes were evaluated in the limited number of studies that met inclusion criteria; this might foreground the potential benefits of considering participants' perspectives in generating a wider range of outcomes which are not only relevant to the intervention but also are consistent with what people living with dementia value. ${ }^{58}$

Although robustly designed evaluation methods/approaches are necessary to determine if an intervention is effective, assessing the outcomes of participatory arts pro- grams (particularly social engagement) for older people living with dementia in the community is challenging. This might be due to the likelihood that evaluation is often tailored to justifying the funding or demonstrating that the provision of art in the health arena is cost-effective, rather than seeking insight into the perceived impacts of such art activities on the participants. Although the arts emphasis is on participation, social interaction, sensation, imagination, and meanings, such factors might be more challenging to test, particularly for those living with dementia; ${ }^{31}$ which supports a need for focus on these elements, particularly social engagement factors, and taking them into consideration when designing, running and evaluating arts programs for those living dementia.

Regarding the evaluation approaches chosen, interview sessions and observations were the preferred evaluation method/approach to assess the outcomes of participatory arts programs. Interview sessions were also reported by participants' to be more desirable as they can chat freely with another person regarding their perspectives rather than addressing a questionnaire. ${ }^{28}$ Comparison of this finding with those of other studies such as Hubbard et al. (2003) confirms the usefulness of interview and observations for privileging the voice of people living with dementia. However, particular care must be taken to account for the potential effect of researcher assumptions, ${ }^{23}$ which was also supported by other studies; $;^{59,60}$ as well as the differences in retold stories of the experience of dementia in interviews with couples when one has a diagnosis of dementia. ${ }^{61}$

Reviewing the suitability of standardized measures for older people living with dementia against rigorous criteria including the conceptual and measurement model, reliability, validity, responsiveness, interpretability, respondent and administrative burden, cultural and language adaptations is strongly recommended. Such evaluation approaches need to be complemented with qualitative approaches that enable the exploration of the perceived effectiveness of participatory art in a more comprehensive way including the social inclusion aspect of participatory arts programs.

As stated by Weitzman and Levkoff (2000), incorporating qualitative and quantitative approaches for minority elders in health studies is useful in ensuring that the quantitative assessments remained culturally relevant and in dealing with some of reliability issues with qualitative data.

Alternative approaches to evidence collection might assist policymakers and community-based arts agencies to compare 
findings and further understand the potential of community-based arts for people living with dementia, particularly in fostering social engagement.

\section{Implications for future research and practice}

Although there are methodological limitations, this scoping review presents a series of findings arguing the importance of the selection of measurement tools/evaluation methods or approaches to assess the outcomes of participatory communitybased arts activities for older people living with dementia. Participatory community arts have been demonstrated to deliver benefits across multiple social parameters. ${ }^{46,62}$

There is a definite need for finding appropriate measurement tools or evaluation methods/ approaches to assess the outcomes of participatory community-based arts programs for older people living with dementia. This may include generating more standardized and tailored scales as well as combining qualitative and quantitative research and considering the opinions, expectations and values of people living with dementia.

\section{Conclusions}

This scoping review was undertaken to investigate the existing measurement tools and evaluation methods/approaches used for assessing the outcomes of participatory arts activities in the community for older people with dementia. The study contributes to our understanding of the impact of participatory community-based arts programs for older people living with dementia.

This would be a fruitful area for further work in order to generate a richer pool of data for available and useful assessment tools to be used by researchers in evaluating the outcomes of community-based arts activities planned, organized and delivered to a wide range of participants in the community including older people with dementia.

Evaluation of community-based arts programs for older people living with dementia should include methods to gain deeper insight into the impacts of arts programs while considering participants' values and perspectives and the social interaction benefits of such programs.

\section{Study limitations}

The main weakness of this study was the limited availability of research on participatory arts programs for older people with dementia in the community. Also, the scope of this study was limited in terms of only discussing the programs for older peo- ple; it would also be useful to extend future research to continue to survey the measurement tools or evaluation methods/approaches and the main outcomes sought on younger people living dementia. Also, the study did not include passive arts programs.

\section{References}

1. Dicker D, Nguyen G, Abate D, et al. Global, regional, and national age-sexspecific mortality and life expectancy, 1950-2017: a systematic analysis for the Global Burden of Disease Study 2017. Lancet 2018;392:1684-735.

2. Livingston G, Sommerlad A, Orgeta V, et al. Dementia prevention, intervention, and care. Lancet 2017;390:2673-734.

3. World Health Organization. Dementia 2017. Available from: https://www.who. int/mental_health/mhgap/dem_slides.pdf

4. World Health Organization. Dementia: a public health priority: World Health Organization; 2012.

5. Wu YT, Clare L, Jones IR, et al. Inequalities in living well with dementia - The impact of deprivation on wellbeing, quality of life and life satisfaction: Results from the improving the experience of dementia and enhancing active life study. Int $\mathrm{J}$ Geriat Psychiat 2018;33:1736-42.

6. Biggs S, Carr A, Haapala I. Dementia as a source of social disadvantage and exclusion. Austral J Ageing 2019;38:26-33.

7. Riley RJ, Burgener S, Buckwalter KC. Anxiety and stigma in dementia: a threat to aging in place. Nursing Clin 2014;49:213-31.

8. Batsch NL, Mittelman MS. Overcoming the stigma of dementia. World Alzheimer Report 2012;1:36-8.

9. Walsh K, Scharf T, Keating N. Social exclusion of older persons: a scoping review and conceptual framework. Eur J Ageing 2017;14:81-98.

10. Kasper JD, Freedman VA, Spillman BC, Wolff JL. The disproportionate impact of dementia on family and unpaid caregiving to older adults. Health Affairs 2015;34:1642-9.

11. Hackett RA, Steptoe A, Cadar D, Fancourt D. Social engagement before and after dementia diagnosis in the English Longitudinal Study of Ageing. PLoS One 2019;14(8).

12. Sun W, Clarke S-L, Madahey H, Zhou P. Recovery intervention to promote social connectedness through social recreational programs for persons with dementia: a critical analysis. advances in dementia research. IntechOpen; 2019.
13. Tsekleves E, Bingley AF, Luján Escalante MA, Gradinar A. Engaging people with dementia in designing playful and creative practices: Co-design or co-creation? Dementia 2020;19: 915-31.

14. Melunsky N, Crellin N, Dudzinski E, et al. The experience of family carers attending a joint reminiscence group with people with dementia: A thematic analysis. Dementia 2015;14:842-59.

15. Innes A, Page SJ, Cutler C. Barriers to leisure participation for people with dementia and their carers: An exploratory analysis of carer and people with dementia's experiences. Dementia 2016;15:1643-65.

16. Stubbs B, Eggermont L, Soundy A, et al. What are the factors associated with physical activity (PA) participation in community dwelling adults with dementia? A systematic review of PA correlates. Archiv Gerontol Geriatr 2014;59: 195-203.

17. Noone S, Jenkins N. Digging for Dementia: Exploring the experience of community gardening from the perspectives of people with dementia. Aging Mental Health 2018;22:881-8.

18. Selkrig M. Learning about ourselves from others: transformation of artists' identities through community-based arts practice. Int J Lifelong Educ 2011;30: 577-89.

19. Richards M, Lawthom R, RunswickCole K. Community-based arts research for people with learning disabilities: challenging misconceptions about learning disabilities. Disabil Soc 2019;34: 204-27.

20. Lagacé M, Briand C, Desrosiers J, Larivière N. A qualitative exploration of a community-based singing activity on the recovery process of people living with mental illness. Br J Occupat Ther 2016;79:178-87.

21. Stickley T, Hui A. Social prescribing through arts on prescription in a UK city: participants' perspectives (part 1). Public Health 2012;126:574-9.

22. Burnside LD, Knecht MJ, Hopley EK, Logsdon RG. here: now - Conceptual model of the impact of an experiential arts program on persons with dementia and their care partners. Dementia 2017;16:29-45.

23. Unadkat S, Camic PM, Vella-Burrows T. Understanding the experience of group singing for couples where one partner has a diagnosis of dementia. Gerontologist 2017;57:469-78.

24. Stickley T, Wright N, Slade M. The art of recovery: outcomes from participatory arts activities for people using mental 
health services. J Mental Health 2018;27:367-73.

25. Castora-Binkley M, Noelker L, Prohaska T, Satariano W. Impact of arts participation on health outcomes for older adults. J Aging Human Arts 2010;4:352-67.

26. Qureshi H. Outcomes in social care practice. Social Policy Research Unit 2001; Volume number 7.

27. Krohn M. Art therapy and the quality of life among elderly with dementia; 2018. Retrieved from https://minds.wisconsin.edu/bitstream/handle/1793/77800/Ar $\mathrm{t} \% 20$ Therapy $\% 20$ and $\% 20$ the $\% 20$ Qualit y\%20of\%20Life\%20among\%20Elderly $\% 20$ with $\% 20$ Dementia.pdf?sequence $=1$ \&isAllowed $=\mathrm{y}$

28. Camic PM, Tischler V, Pearman $\mathrm{CH}$. Viewing and making art together: a multi-session art-gallery-based intervention for people with dementia and their carers. Aging Mental Health 2014;18: 161-8.

29. Lyons S, Karkou V, Roe B, et al. What research evidence is there that dance movement therapy improves the health and wellbeing of older adults with dementia? A systematic review and descriptive narrative summary. Arts Psychother 2018;60:32-40.

30. Windle G, Algar-Skaife K, Caulfield M, et al. Enhancing communication between dementia care staff and their residents: an arts-inspired intervention. Aging Mental Health 2019:1-10.

31. Zeilig H, Killick J, Fox C. The participative arts for people living with a dementia: a critical review. Int J Ageing Later Life 2014;9:7-34.

32. Lee R, Wong J, Shoon WL, et al. Art therapy for the prevention of cognitive decline. Arts Psychother 2019;64:20-5.

33. Young R, Camic PM, Tischler V. The impact of community-based arts and health interventions on cognition in people with dementia: A systematic literature review. Aging Mental Health 2016;20:337-51.

34. Pickett J, Bird C, Ballard C, et al. A roadmap to advance dementia research in prevention, diagnosis, intervention, and care by 2025 . Int J Geriatr Psychiat 2018;33:900-6.

35. Gargon E, Gurung B, Medley N, et al. Choosing important health outcomes for comparative effectiveness research: a systematic review. PLoS One 2014;9(6).

36. Sinha I, Jones L, Smyth RL, Williamson PR. A systematic review of studies that aim to determine which outcomes to measure in clinical trials in children. PLoS Med 2008;5(4).

37. Moniz-Cook E, Vernooij-Dassen M, Woods R, et al. A European consensus on outcome measures for psychosocial intervention research in dementia care. Aging Mental Health 2008;12:14-29.

38. Smith S, Lamping D, Banerjee S, et al. Measurement of health-related quality of life for people with dementia: development of a new instrument (DEMQOL) and an evaluation of current methodology. Health Technol Assess (Winchester, England) 2005;9:1-93.

39. Ward MC, Milligan C, Rose E, et al. The benefits of community-based participatory arts activities for people living with dementia: a thematic scoping review. Arts Health 2020:1-27.

40. Levac D, Colquhoun H, O'Brien KK. Scoping studies: advancing the methodology. Implement Sci 2010;5:69.

41. Makhoul J, Chehab RF, Shaito Z, Sibai AM. A scoping review of reporting 'Ethical Research Practices' in research conducted among refugees and waraffected populations in the Arab world. BMC Med Ethics 2018;19:36.

42. Arksey H, O’Malley L. Scoping studies: towards a methodological framework. Int J Social Res Methodol 2005;8:19-32.

43. Beard RL. Art therapies and dementia care: A systematic review. Dementia 2012;11:633-56.

44. Letts L, Wilkins S, Law M, et al. Critical review form-qualitative studies (version 2.0); 2007. Available from: https://www. unisa.edu.au/contentassets/72bf75606a2 b4abcaf7f17404af374ad/7b-mcmasters_qualreview_version2-01.pdf

45. Camic PM, Hulbert S, Kimmel J. Museum object handling: A health-promoting community-based activity for dementia care. J Health Psychol 2019;2 4:787-98.

46. Schall A, Tesky VA, Adams A-K, Pantel J. Art museum-based intervention to promote emotional well-being and improve quality of life in people with dementia: The ARTEMIS project. Dementia 2018;17:728-43.

47. Ullán AM, Belver MH, Badía M, et al. Contributions of an artistic educational program for older people with early dementia: An exploratory qualitative study. Dementia 2013;12:425-46.

48. Wieczorkiewicz A, Hooper-Greenehill E. Museums and the Shaping of Knowledge, E. Hooper-Greenehill, Routledge, London and New York 1993:[recenzja]. Etnografia Polska. 1997;41(1-2).

49. Kugler P, Turvey M, Malloy $\mathrm{T}$, et al. Steps to an ecology of mind. Chicago: University of Chicago Press; 2000.

50. Cohen GD, Perlstein S, Chapline J, et al. The impact of professionally conducted cultural programs on the physical health, mental health, and social functioning of older adults. The Gerontologist 2006;46:726-34.

51. Larner A. Addenbrooke's Cognitive Examination (ACE) for the diagnosis and differential diagnosis of dementia. Clin Neurol Neurosur 2007;109:491-4.

52. Kaufer DI, Cummings JL, Ketchel P, et al. Validation of the NPI-Q, a brief clinical form of the Neuropsychiatric Inventory. J Neuropsychiat Clin Neurosci 2000;12:233-9.

53. Johnson J, Culverwell A, Hulbert S, et al. Museum activities in dementia care: Using visual analog scales to measure subjective wellbeing. Dementia 2017;16: 591-610.

54. Kuemmel A, Haberstroh J, Pantel J. CODEM instrument: developing a tool to assess communication behavior in dementia. GeroPsych 2014;27:23-31.

55. Bygren LO, Konlaan BB, Johansson SE. Attendance at cultural events, reading books or periodicals, and making music or singing in a choir as determinants for survival: Swedish interview survey of living conditions. BMJ 1996;313:1577-80.

56. Glass TA, De Leon CM, Marottoli RA, Berkman LF. Population based study of social and productive activities as predictors of survival among elderly Americans. BMJ 1999;319:478-83.

57. Beauchet O, Bastien T, Mittelman M, et al. Participatory art-based activity, community-dwelling older adults and changes in health condition: Results from a pre-post intervention, single-arm, prospective and longitudinal study. Maturitas 2020 [Epub ahead of print].

58. Harding AJ, Morbey H, Ahmed F, et al. What is important to people living with dementia?: the 'long-list' of outcome items in the development of a core outcome set for use in the evaluation of nonpharmacological community-based health and social care interventions. BMC Geriatrics 2019;19:94

59. Bond J, Corner L. Researching dementia: are there unique methodological challenges for health services research? Ageing Soc 2001;21:95-116.

60. Moore TF, Hollett J. Giving voice to persons living with dementia: The researcher's opportunities and challenges. Nursing Sci Q 2003;16:163-7.

61. Quinn C. Conducting interviews with people with dementia and their caregivers. SAGE Publications Ltd; 2017.

62. Phinney A, Moody EM, Small JA. The effect of a community-engaged arts program on older adults' well-being. Canad J Aging 2014;33:336-45. 